\title{
Chronic Subdural Hematoma in Elderly Patient with EDTA-dependent Pseudothrombocytopenia Recently Treated with Aspirin and Warfarin: Case Report
}

\author{
Masato TOSA, ${ }^{1}$ Hiroshi FujITA, ${ }^{2}$ Yumiko ISHIHAmA, ${ }^{3}$ Shigeko NiSHIMURA, ${ }^{2}$ \\ and Takafumi IDE ${ }^{1}$
Departments of ${ }^{1}$ Neurosurgery, ${ }^{2}$ Transfusion Medicine, and ${ }^{3}$ Clinical Laboratory, Tokyo Metropolitan Bokutoh Hospital, Tokyo

\begin{abstract}
A 78-year-old man who had a history of myocardial and cerebral infarction and who was treated with aspirin and warfarin, presented with left chronic subdural hematoma. Cerebral computed tomography showed severe brain compression of hematoma with midline shift, indicating the need for emergent surgery. The hematology and clotting tests upon admission revealed severe thrombocytopenia (platelet count, $1.3 \times 10^{4} / \mu \mathrm{L}$ ) with normal clotting activity. Because platelet aggregation was evident in the smear, we re-examined the patient for hematology using tubes that contained heparin, showing also low platelet count $\left(2.3 \times 10^{4} / \mu \mathrm{L}\right)$. The day on admission, we performed irrigation and drainage of the chronic subdural hematoma through single burr-hole craniostomy. During surgery, we used 10 units of platelet concentrates (PCs) for the reason that the patient was taking aspirin and coagulopathy derived from low platelet count could not be excluded. After surgery, we re-evaluated the hematology of the blood stored in tubes that contained ethylenediaminetetraacetic acid (EDTA) with or without kanamycin (KM). Treatment with KM dissociated EDTA-induced platelet aggregation and revealed platelet counts with highest accuracy (no KM treatment, $1.3 \times 10^{4} / \mu \mathrm{L} ; \mathrm{KM}$ treatment, $15.2 \times 10^{4} / \mu \mathrm{L}$ ). This phenomenon is called EDTA-Dependent Pseudothrombocytopenia (PTCP) defined as falsely low platelet counts reported by automated hematology analyzers due to platelet aggretgation. Awareness of the phenomenon will enable neurosurgeons to manage patients with PTCP appropriately and clinical laboratory especially in emergency hospital is recommended to prepare for the hematological tubes being added KM in routine analysis, resulting in preventing mistaken diagnosis.
\end{abstract}

Key words: pseudothrombocytopenia, kanamycin, chronic subdural hematoma, aspirin

\section{Introduction}

Thrombocytopenia associated with intracranial bleeding is typically severe; the platelet threshold for transfusion of platelet concentrates (PC) is $7.0 \times 10^{4} / \mu \mathrm{L}$ in Japan. ${ }^{1)}$ In the elderly, anti-thrombotic agents such as aspirin and warfarin are used to treat previous occurrences of myocardial and cerebral infarction. In our hospital's emergency department, we often use anti-thrombotic agents to treat patients with complications related to intracranial bleeding. Some reports support the strategic use of PC transfusion in patients who had used anti-platelet drugs recently. ${ }^{2,3)}$ For patients who used aspirin or adenosine receptor antagonists such as clopidogrel, PC transfusion

Received August 1, 2013; Accepted September 20, 2013 was deemed potentially necessary during certain types of neurosurgery such as urgent hematoma exclusion. For elective surgery, antithrombotic agents were stopped 7 to 10 days before surgery to reduce the risk of bleeding. ${ }^{3,4)}$

During PC transfusion treatment, we experienced difficulties in managing patients with pseudothrombocytopenia (PTCP), ${ }^{5}$ and determining the cause of PTCP effectively delayed elective surgery. ${ }^{6,7)}$ A state of PTCP is defined as falsely low platelet counts reported by automated hematology analyzers due to platelet aggregation. Commonly, this clumping is caused by alterations of platelet surface glycoproteins when they are incubated with a calcium chelator such as ethylenediaminetetraacetic acid (EDTA). The modified platelet antigens react with anti-platelet autoantibodies to form large agglutinates. ${ }^{8,9}$ Some resources state that platelet aggregation in patients with EDTA-dependent PTCP can be prevented by using 
other anticoagulants such as sodium citrate or heparin, but we found that these agents can induce platelet clumping too, and thus result in spuriously low platelet counts. ${ }^{9)}$ In emergency, unnecessary platelet concentrates are sometimes transfused for the patients with PTCP. ${ }^{10,11)}$ To avoid unnecessary treatments, the addition of aminoglycosides, such as kanamycin (KM) or amikacin, has also been indicated as a useful method to diagnose EDTA-induced PTCP and evaluate patients appropriately in routine testing. ${ }^{12)}$

We have attended to an elderly patient whose recent aspirin use had complicated its conditions of PTCP and symptomatic chronic subdural hematoma, and we carefully considered using PC transfusion in this patient during neurosurgery. In this paper, we present this case study and the relationships among PC transfusion, aspirin use, and PTCP.

\section{Case Report}

A 78-year-old man complained of sudden disturbance in gait. He had a history of myocardial and cerebral infarction, for which he was being treated with aspirin $(81 \mathrm{mg} /$ day) and warfarin (1 mg/day). He was transferred to our hospital for urgent surgery because a left subdural hematoma was observed in his head on computed tomography (CT). The hematology and clotting tests performed upon admission revealed severe thrombocytopenia (platelet count, $1.3 \times 10^{4} / \mu \mathrm{L}$ ) with normal clotting activity [prothrombin time (PT), 90.4\%; activated partial PT (aPTT), 24.5 seconds]. We considered the possibility that warfarin was not addressing his clotting activity effectively. However, we observed no anemia in the patient (hemoglobin, $13.6 \mathrm{~g} / \mathrm{dL}$; hematocrit, 40.1\%). We noted mild hepatic dysfunction (aspartate aminotransferase, 38 IU/L; alanine aminotransferase, $39 \mathrm{IU} / \mathrm{L}$ ) and normal renal function. Because peripheral blood smears revealed platelet clumping (Fig. 1A), we re-examined the hematology following blood collection in tubes that contained heparin. In this case, the platelet counts were $2.3 \times 10^{4} / \mu \mathrm{L}$, and the blood smear still showed platelet clumping (Fig. 1B). We were unable to examine the platelet counts, further or promptly, for accuracy before performing the emergent operation. Since cerebral CT scan revealed old cerebral infarction in right middle cerebral artery territory and left chronic subdural hematoma with severe brain compression and midline shift (Fig. 2A), we performed evacuation of the hematoma through single burr-hole craniostomy. During surgery, we included 10 units of platelet concentrates (PCs) to reduce the bleeding risk in patients who were recently treated with anti-platelet drugs. Methods for evaluating platelet aggregation were not available in our laboratory. The total blood loss during surgery was within several milliliters. The day after surgery, we evaluated blood that had been stored in a tube with EDTA, and KM (Meiji Seika Pharma Co., Ltd., Tokyo) was added into the tube (final concentration: $20 \mathrm{mg} / \mathrm{mL}$ ). Treatment with KM dissociated the EDTA-induced platelet clumping and revealed accurate platelet counts (without KM, $1.3 \times$ $10^{4} / \mu \mathrm{L}$; with $\mathrm{KM}, 15.2 \times 10^{4} / \mu \mathrm{L}$ ) (Fig. 1C). Since we obtained accurate platelet counts which were within
A
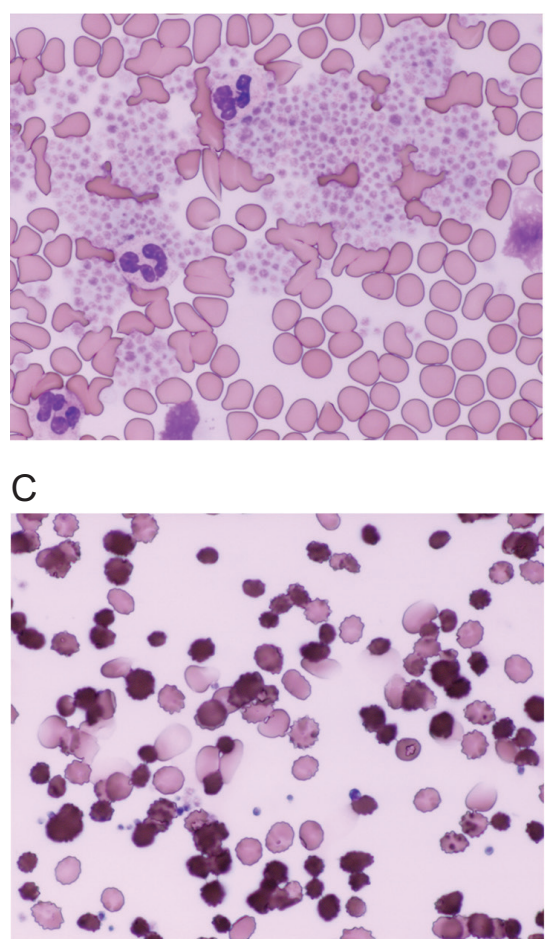

B

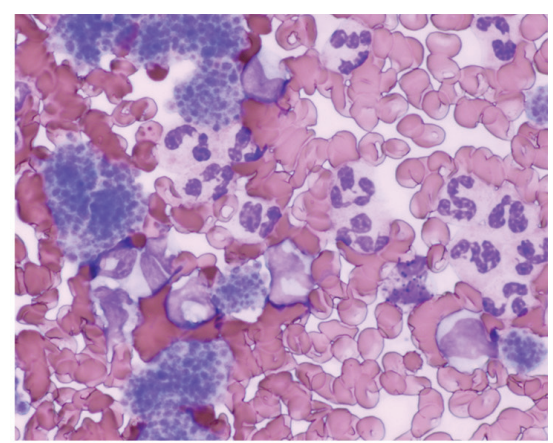

D

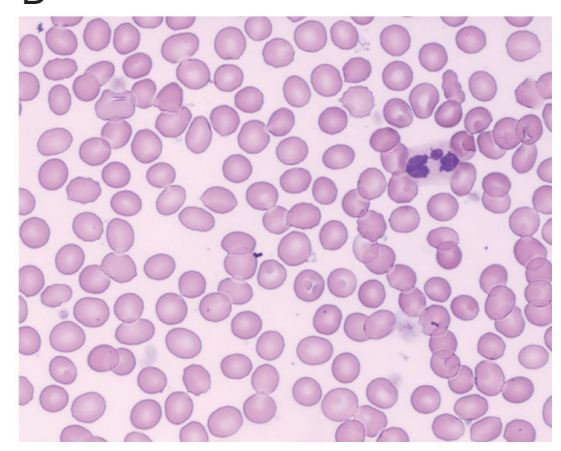

Fig. 1 Peripheral blood smears following storage in tubes with one of several anticoagulants. A: Ethylenediaminetetraacetic acid (EDTA); peripheral-blood smear (May-Giemsa stain, 400× magnification) shows platelet clumping at admission. An electronic counting machine was used to generate an estimated platelet count of $1.3 \times 10^{4} / \mu \mathrm{L}$. These findings were also noted similarly after surgery. B: Heparin; peripheral-blood smear (May-Giemsa stain, 400× magnification) shows platelet clumping at admission. An electronic counting machine was used to generate an estimated platelet count of $2.3 \times 10^{4} / \mu \mathrm{L}$. These findings were also noted similarly after surgery. C: EDTA with kanamycin; a peripheral-blood smear (May-Giemsa stain, 400× magnification) showed dissolution of platelet clumping after surgery. An electronic counting machine was used to generate an estimated platelet count of $15.2 \times 10^{4} / \mu \mathrm{L}$. D: Citrate sodium; a peripheral-blood smear (May-Giemsa stain, 400× magnification) showed no platelet clumping after surgery. An electronic counting machine was used to generate an estimated platelet count of $16.9 \times 10^{4} / \mu \mathrm{L}$. 
normal limits from KM-treated blood, we could avoid drawing more blood and additional PC transfusion postoperatively. Post-operative CT scans of the head revealed no bleeding (Fig. 2B).

Seven days after the surgery, we examined blood hematology after it had been stored in various anticoagulants (EDTA, heparin, or citrate sodium). We found lower platelet counts (PTCP) in tubes that contained EDTA or heparin compared to tubes with citrate sodium (Table 1 and Fig. 1A, B, D). The addition of KM allowed us to obtain more accurate platelet counts in blood stored in
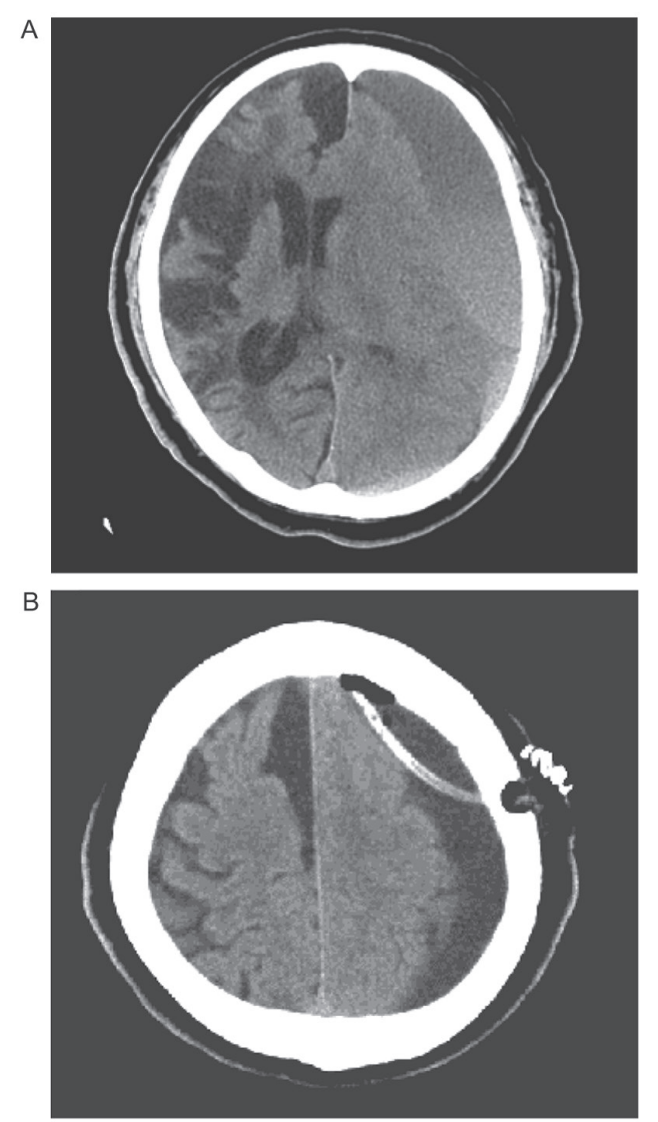

Fig. 2 Head computed tomography before (A) and after (B) surgery.

Table 1 Estimated numbers of platelets in the peripheral blood stored for 7 days after surgery in tube with one of several anticoagulants

\begin{tabular}{lcc}
\hline Anticoagulants & $\begin{array}{c}\text { Platelet counts } \\
\left(\times 10^{4} / \mu \mathrm{L}\right) \text { without } \\
\text { kanamycin }\end{array}$ & $\begin{array}{c}\text { Platelet counts } \\
\left(\times 10^{4} / \mu \mathrm{L}\right) \text { with } \\
\text { kanamycin }\end{array}$ \\
\hline EDTA & 2.9 & 18.1 \\
Heparin & 7.0 & 16.6 \\
Citrate sodium & 16.9 & 18.1 \\
\hline
\end{tabular}

EDTA: ethylenediaminetetraacetic acid. tubes with EDTA or heparin (Table 1).

After the patient demonstrated improvements in his gait, he was transferred to his family hospital for recovery. Warfarin, which was discontinued at admission, was restarted in the seventh day after surgery, and aspirin was discontinued until the transferred day.

\section{Discussion}

I. PC transfusion for neurosurgical patients recently treated with anti-thrombolytic agents such as aspirin or clopidogrel

In Japan, no guidelines have been issued regarding PC transfusion for patients recently treated with antithrombolytic agents. ${ }^{1)}$ The pre-injury use of aspirin and clopidogrel has been associated with increased mortality, and in other countries, there are a number of guidelines regarding neurosurgery on patients recently treated with anti-thrombolytic agents., ${ }^{2,3)}$ However, general hospitals in Japan have no system for evaluating the inhibitory effects of anti-platelet agents on platelet function. These guidelines are needed to enable the neurosurgeon to select the best treatment for patients who have developed intracranial bleeding and have used anti-thrombolytic agents recently. In our case, an elderly patient who had been using aspirin and warfarin presented with gait disturbance due to chronic subdural hematoma. Because clotting tests were normal, we performed no reversal for warfarin. We stopped warfarin and did not use vitamin $\mathrm{K}$ or fresh frozen plasma. Campbell et al. reported that patients with intracranial bleeding such as spontaneous cerebral bleeding and traumatic brain injury who used aspirin recently should be indicated for PC transfusion. ${ }^{2)}$ However, neither Campbell's paper nor the Japanese guidelines ${ }^{1)}$ provided comments on chronic subdural hematoma. ${ }^{2)}$ PC transfusion was performed during the urgent surgery for chronic subdural hematoma with idiopathic thrombocytopenic purpura. ${ }^{13)}$ In Japan, the elderly population and usage of anti-platelet agents (e.g., aspirin and clopidogrel) have increased. We believe domestic guidelines regarding strategic treatment for patients with intracranial bleedings who have used anti-thrombolytic agents recently are necessary. We hope that Japan's Neurosurgical Society will develop these domestic guidelines in future.

\section{Pre-surgery pseudothrombocytopenia}

Various studies on PTCP and surgery have been reported. ${ }^{10,14-16)}$ However, few studies have been published on neurosurgery and PTCP. ${ }^{17)}$ When PTCP is noted prior to surgery, neurosurgeons are required to obtain accurate platelet counts. When EDTA-dependent PTCP is suspected, we first challenge the hematology again using venipuncture tubes containing the other anticoagulants such as heparin, citrate, and so on. ${ }^{9,18-20)}$ Measurement should be immediately performed ${ }^{19)}$ and temperatures during 
the measurement are very sensitive in each patient,,9,20,21) because optimal temperatures of autoantibodies are different among the patients.

On the other hand, the addition of KM is very useful for obtaining accurate platelet counts without the notice of the measurement time and temperatures. ${ }^{12)}$ In this case, we determined accurate platelet counts in EDTA- and heparin-induced PTCP the day after the operation. In emergency situations, the addition of KM to hematological tubes for use in routine analysis is easy and allows for accurate counts immediately.

In our hospital, KM typically is absent in the clinical laboratory, and thus, we could not respond to the PTCP until after the urgent surgery. In the future, KM will be added to hematological tubes routinely for evaluation of PTCP in our laboratory.

\section{Conclusion}

In this case report, we demonstrated that recent aspirin and warfarin use complicated the patient's chronic subdural hematoma. We discussed the relationship among PC, anti-thrombolytic agents, and PTCP.

\section{Conflicts of Interest Disclosure}

All authors have no interests to declare.

\section{References}

1) Ministry of Health, Labour and Welfare: Ketsueki-seizai no shiyou-shishin, ed 4. Tokyo, Japan Red Cross, 2012 (Japanese)

2) Campbell PG, Sen A, Yadla S, Jabbour P, Jallo J: Emergency reversal of antiplatelet agents in patients presenting with an intracranial hemorrhage: a clinical review. World Neurosurg 74: 279-285, 2010

3) Levi MM, Eerenberg E, Löwenberg E, Kamphuisen PW: Bleeding in patients using new anticoagulants or antiplatelet agents: risk factors and management. Neth J Med 68: 68-76, 2010

4) Merriman E: Antiplatelet drugs, anticoagulants and elective surgery. Aust Prescr 34: 139-149, 2011

5) Sane DC, Damaraju LV, Topol EJ, Cabot CF, Mascelli MA, Harrington RA, Simoons ML, Califf RM: Occurrence and clinical significance of pseudothrombocytopenia during abciximab therapy. J Am Coll Cardiol 36: 75-83, 2000

6) Skues MA, Richards MJ, Jarvis AP, Prys-Roberts C: Preinduction atropine or glycopyrrolate and hemodynamic changes associated with induction and maintenance of anesthesia with propofol and alfentanil. Anesth Analg 69: 386-390, 1989

7) Wilkes NJ, Smith NA, Mallett SV: Anticoagulant-induced pseudothrombocytopenia in a patient presenting for coronary artery bypass grafting. Br J Anaesth 84: 640-642, 2000

8) Bizzaro N: EDTA-dependent pseudothrombocytopenia: a clinical and epidemiological study of 112 cases, with 10-year follow-up. Am J Hematol 50: 103-109, 1995
9) Casonato A, Bertomoro A, Pontara E, Dannhauser D, Lazzaro AR, Girolami A: EDTA dependent pseudothrombocytopenia caused by antibodies against the cytoadhesive receptor of platelet gpIIB-IIIA. J Clin Pathol 47: 625-630, 1994

10) Kocum TH, Katircibasi TM, Sezgin AT, Atalay H: An unusual cause of mismanagement in an acute myocardial infarction case: pseudothrombocytopenia. Am J Emerg Med 26: 740. e1-e2, 2008

11) Lau LG, Chng WJ, Liu TC: Transfusion medicine illustrated. Unnecessary transfusions due to pseudothrombocytopenia. Transfusion 44: 801, 2004

12) Sakurai S, Shiojima I, Tanigawa T, Nakahara K: Aminoglycosides prevent and dissociate the aggregation of platelets in patients with EDTA-dependent pseudothrombocytopenia. Br J Haematol 99: 817-823, 1997

13) Panicker JN, Pavithran K, Thomas M: Management of subdural hematoma in immune thrombocytopenic purpura: report of seven patients and a literature review. Clin Neurol Neurosurg 111: 189-192, 2009

14) Abe H, Shimizu T, Cho H, Kubota Y, Umeda T, Kurumi Y, Tani T: Occult breast cancer with EDTA-dependent pseudothrombocytopenia-a case report. Gan To Kagaku Ryoho 37: 915-918, 2010 (Japanese)

15) Kim HJ, Moh IH, Yoo H, Son S, Jung DH, Lee HG, Han DH, Park JH, Kim HS, Kim JH: Ethylenediaminetetraacetic acid-dependent pseudothrombocytopenia associated with neuroendocrine carcinoma: a case report. Oncol Lett 4: 86-88, 2012

16) Nair SK, Shah R, Petko M, Keogh BE: Pseudothrombocytopenia in cardiac surgical practice. Interact Cardiovasc Thorac Surg 6: 565-566, 2007

17) Yamaguchi M, Mayumi M, Kasuya T: [A patient with EDTAdependent pseudothrombocytopenia who underwent emergent clipping surgery for a ruptured aneurysm]. Masui 47: 57-59, 1998 (Japanese)

18) Nishioka T, Yokota M, Tsuda I, Tatsumi N: Flow cytometric analysis of platelet activation under calcium ion-chelating conditions. Clin Lab Haematol 24: 115-119, 2002

19) Yoshikawa T, Nakanishi K, Maruta T, Takenaka D, Hirota S, Matsumoto S, Saigo K, Ohno Y, Fujii M, Sugimura K: Anticoagulant-induced pseudothrombocytopenia occurring after transcatheter arterial embolization for hepatocellular carcinoma. Jpn J Clin Oncol 36: 527-531, 2006

20) Zandecki M, Genevieve F, Gerard J, Godon A: Spurious counts and spurious results on haematology analysers: a review. Part I: platelets. Int J Lab Hematol 29: 4-20, 2007

21) Lippi G, Plebani M: EDTA-dependent pseudothrombocytopenia: further insights and recommendations for prevention of a clinically threatening artifact. Clin Chem Lab Med 50: 1281-1285, 2012

Address reprint requests to: Hiroshi Fujita, $\mathrm{MD}, \mathrm{PhD}$, Department of Transfusion Medicine, Tokyo Metropolitan Bokutoh Hospital, 4-23-15 Koutoubashi, Sumida-ku, Tokyo 130-8575, Japan. e-mail: yuketsuka@bokutoh-hp.metro.tokyo.jp 